\title{
Scheduled Activity Ideal Date Range
}

National Cancer Institute

\section{Source}

National Cancer Institute. Scheduled Activity Ideal Date Range. NCI Thesaurus. Code C94075.

The date and time span specifying when the activity was orig inally scheduled to begin and end. 